Artículo

\title{
Productividad del agua y rendimiento de maíz bajo diferente disponibilidad de humedad
}

\author{
Homero Alonso-Sánchez ${ }^{1}$ \\ Margarita Tadeo-Robledo ${ }^{1 \S}$ \\ Alejandro Espinosa-Calderón ${ }^{2}$ \\ Job Zaragoza-Esparza ${ }^{1}$ \\ Consuelo López-López ${ }^{1}$
}

\begin{abstract}
${ }^{1}$ Ingeniería Agrícola de la Facultad de Estudios Superiores Cuautitlán-UNAM. Carretera CuautitlánTeoloyucan km 2.5, Col. San Sebastián Xhala, Cuautitlán Izcalli, Estado de México, México. CP. 54700. (Alonso_m77@ @otmail.com; jobzaragoza4929@yahoo.com; lopez8con@gmail.com). $\quad{ }^{2}$ Campo Experimental Valle de México-INIFAP. Carretera Los Reyes-Texcoco km 13.5, Coatlinchán, Texcoco, Estado de México, México. CP. 56250. (espinoale@yahoo.com.mx).

${ }^{\S}$ Autora para correspondencia: tadeorobledo@yahoo.com.
\end{abstract}

\section{Resumen}

La experimentación con híbridos para condiciones con escasez de agua es fundamental para la seguridad hídrica y alimentaria, integrando el manejo de cultivos con el de los recursos hídricos. El objetivo en este trabajo fue definir la productividad del agua, el rendimiento de grano y el peso hectolítrico en tres ambientes con diferente disponibilidad de humedad: riego y temporal en la FESC-UNAM y riego en Texcoco, Estado de México; utilizando cinco híbridos de maíz de la UNAM y cinco del INIFAP, con dos densidades de siembra. Se estableció el experimento en bloques completos al azar y se registraron variables durante el ciclo y a la cosecha; la productividad total del agua se calculó como el volumen total de agua entre el rendimiento de grano a $14 \%$ de humedad. Utilizando SAS se realizó el ANOVA para el análisis de los factores y sus interacciones, se separaron las medias con la prueba de Tukey al 5\%. Se encontró efecto de la interacción ambiente $\mathrm{x}$ híbrido e híbrido x densidad de siembra en la productividad total del agua. En el rendimiento de grano se encontró efecto de la interacción ambiente x híbrido, ambiente x densidad e híbrido x densidad. Se encontró efecto altamente significativo en el peso hectolítrico del ambiente y del tipo de híbrido. La interacción del híbrido Atziri Puma con los ambientes y con la densidad de siembra mostraron los valores máximos de productividad total del agua y del rendimiento de grano.

Palabras clave: eficiencia en el uso del agua, punta de riego, riego más precipitación, riego y temporal, volumen bruto.

Recibido: enero de 2020

Aceptado: abril de 2020 


\section{Introducción}

El uso eficiente del agua en la agricultura se interpreta como el alcance de los rendimientos máximos utilizando la misma cantidad o menos que en años anteriores. Esto es importante, ya que las estadísticas indican que el sector agrícola consume $77 \%$ de agua dulce, siendo también una actividad que contamina las corrientes superficiales y subterráneas. En ese contexto el panorama de la producción de alimentos en México debe alinearse al uso eficiente del agua.

En México, la Universidad Nacional Autónoma de México (FESC-UNAM) y el Instituto Nacional de Investigaciones Forestales Agrícolas y Pecuarias (INIFAP), han sumado esfuerzos en mejoramiento genético de maíz para generar híbridos a partir de materiales nativos y maíces mejorados, y desarrollar materiales con alto potencial de rendimientos en condiciones de baja disponibilidad de humedad.

La UNAM e INIFAP trabajan desde 1992 a la fecha en inclusión de la androesterilidad como una alternativa para facilitar la producción de semillas (Tadeo et al., 2014a), bajo ese esquema se liberó comercialmente en 2011 el híbrido H-51 AE (Espinosa et al., 2012), también se han generado otros maíces híbridos, H-49 AE y H-47 AE, con la misma característica de androesterilidad (Espinosa et al., 2008; Espinosa et al., 2018; Espinosa et al., 2019).

Como una alternativa a la agricultura tradicional y de subsistencia, los híbridos H-50 y H-51 AE, se han utilizado por años en los Valles Altos del altiplano mexicano (González et al., 2007; González et al., 2008). En estos materiales no se encuentran investigaciones recientes sobre la productividad del agua. La productividad del agua se ha manejado con la eficiencia a nivel parcelario y fisiológico (Lamm, 2001) como el rendimiento de grano entre el total de agua utilizada.

Sin embargo, la eficiencia comprende la cantidad de agua que queda almacenada en la zona de raíces con relación a la que sale de la fuente de abastecimiento comprendiendo implícitamente las pérdidas, siendo un parámetro adimensional que no tiene unidades. En este sentido, la productividad se refiere a la cantidad de agua servida en la parcela con relación al rendimiento, la cual es afectada por el método de riego y comprende la eficiencia fisiológica expresándose en unidades de masa por unidad de volumen consumido.

En el caso del maíz, la FAO (2002) indica que el consumo de agua por ciclo está entre 500 y 800 mm y es el más productivo en términos de materia seca comparado con otros granos. El INIFAP (1988) indica láminas de $540 \mathrm{~mm}$ para variedades precoces y $620 \mathrm{~mm}$ para variedades tardías. Los estudios sobre productividad del agua tienen mayor impacto donde la fuente es de acuíferos, como es el caso de las zonas áridas y semiáridas de los Valles Altos (Zamora et al., 2011).

Mencionan que la escasez de agua y la variación interanual de la precipitación son las principales características de estas regiones, en donde, de acuerdo con la FAO-AGL (2002) se ha identificado una productividad del maíz de 0.8 a $1.6 \mathrm{~kg} \mathrm{~m}^{-3}$. Para el uso racional del agua los administradores del recurso requieren conocer su productividad a través de indicadores cuantitativos que permitan generar acciones para las prácticas sostenibles. Para las condiciones del territorio mexicano no existe suficiente información sobre la productividad total del agua para evaluar si los rendimientos están en correspondencia con la cantidad de agua utilizada para riego. 
Este indicador es de vital importancia en el panorama actual de escasez del recurso y ante las políticas públicas donde el balance del agua está impactando como un indicador de peso en la economía, bajo este panorama es urgente conocer los indicadores para los híbridos que se utilizan por región. El objetivo del experimento consistió en identificar la productividad total del agua y las principales variables de rendimiento en tres condiciones diferentes de disponibilidad de humedad (ambientes) de 10 híbridos de maíz blanco en dos densidades de siembra, para contribuir con las bases para las acciones futuras en el uso eficiente del agua en el marco de la agricultura sostenible.

\section{Materiales y métodos}

El experimento se desarrolló bajo tres ambientes relacionados a la disponibilidad de humedad; dos de ellos diferenciados por el manejo bajo punta de riego (FESC-UNAM riego) con fecha de siembra del 07 de junio de 2018 y el otro bajo temporal o secano (FESC-UNAM temporal) con fecha de siembra el 12 de junio del mismo año, dirigidos en la Facultad de Estudios Superiores Cuautitlán de la UNAM (FESC-UNAM), en Cuautitlán Izcalli, Estado de México (latitud 1941' 49" y longitud $\left.-99^{\circ} 41^{\prime} 36^{\prime \prime}\right)$ a la elevación de $2250 \mathrm{msnm}$, perteneciente a una zona con precipitación media normal de $705.4 \mathrm{~mm}$ y temperatura media normal de $15.3^{\circ} \mathrm{C}$ según el Servicio Meteorológico Nacional (SMN, 2017).

El tercer ambiente con riegos de auxilio se implementó el 08 de junio de 2018 en el Ejido de San Luis Huexotla, en el Municipio de Texcoco, Estado de México (latitud 19²9’ 29” y longitud $-98^{\circ}$ 54' 29") a la elevación de 2244 msnm con precipitación media normal de $618 \mathrm{~mm}$. Se emplearon cinco híbridos trilineales de maíz del INIFAP (Espinosa et al., 2018; Espinosa et al., 2019) H-47 AE, H-45 AE, H-53 AE, H-51 AE cuyas semillas se obtuvieron utilizando el esquema de la androesterilidad; también se utilizó el híbrido fértil H-50 de polinización libre y cinco híbridos generados por la UNAM (Tadeo et al., 2016a), Tsíri Puma, Tlaoli Puma, Atziri Puma, Ixim Puma y Cuxi Puma en éstos la obtención de semilla fue a través de cruzas.

Los tratamientos estuvieron conformados por la evaluación de los tres ambientes (factor A); 10 híbridos de maíz (factor B) y dos densidades de siembra (factor C). Los tratamientos se implementaron en unidades experimentales (UE) uniformes en cada ambiente con un diseño en cuatro bloques completos al azar, la UE o parcela útil consistió en un surco de $5 \mathrm{~m}$ de largo por 0.8 $\mathrm{m}$ de ancho.

La preparación del terreno en los ambientes consistió en nivelación para uniformizar la pendiente y beneficiar el riego, un paso del arado de discos, dos pasos de rastra cruzada y el paso de la surcadora para conformar los surcos con $0.8 \mathrm{~m}$ de separación, en esta última se aprovechó para aplicar la fertilización edáfica con la fórmula N P K 80-40-00, se utilizaron urea y fosfato diamónico como fuentes de fertilización en los tres ambientes.

La siembra se realizó manualmente con pala y empleando el sistema punta de riego convencional en el ambiente FESC-UNAM riego y riego de germinación en Texcoco, en el caso del ambiente FESC-UNAM temporal (secano), la humedad de siembra correspondió a la precipitación pluvial. En cada UE se distribuyeron uniformemente 50 semillas en la distancia de $5 \mathrm{~m}$, después de la emergencia de la plántula se ajustó la densidad a 65000 plantas ha $^{-1}$ y 80000 plantas ha ${ }^{-1}$ según correspondiese. 
El control de malezas se realizó con el método químico en el que, con el suelo húmedo, se aplicaron productos en preemergencia, sus ingredientes activos consistieron en: Picloram 2,4 D-amina ( $2 \mathrm{~L}$ $\left.\mathrm{ha}^{-1}\right)$ y Atrazina $\left(2 \mathrm{~kg} \mathrm{ha}^{-1}\right)$ como sellador para hoja ancha. A los 40 días después de la siembra (DDS) se aplicó nuevamente el control químico con, Picloram 2-4D-amina (2 L ha ${ }^{-1}$ ) y Atrazina (2 $\mathrm{kg} \mathrm{ha}^{-1}$ ) para hoja ancha y Nicosulfuron $\left(1.5 \mathrm{~L} \mathrm{ha}^{-1}\right)$ para control de malezas de hoja angosta.

La cosecha se realizó de forma manual en la segunda semana de diciembre de 2018 recolectando manualmente todas las mazorcas de cada UE para obtener el peso completo (PC). De cada UE, 240 en total, se seleccionaron cinco con características comerciales y sanidad adecuada. Esta muestra se utilizó para identificar las variables de productividad y rendimiento.

Con la muestra de cinco mazorcas, se midieron en laboratorio; la longitud de mazorca (LM), las hileras por mazorca $(\mathrm{HM})$, el número de granos por hilera $(\mathrm{GH})$, el peso de grano $(\mathrm{PG})$, la densidad del grano (DG), la humedad del grano (HG), el peso hectolítrico $(\mathrm{PH})$ y el peso de 200 granos. Se calculó el porcentaje de grano $(\% \mathrm{G})$, el número de granos por mazorca $(\mathrm{GM})$, el porcentaje de materia seca (MS) y el rendimiento de grano (RG) con la expresión adaptada de Espinosa et al. (2012): $\mathrm{RG}=(\mathrm{PCxMSxG}) \times \mathrm{FCx} 1.14$.

Donde: $\mathrm{PC}=$ peso completo del total de las mazorcas cosechadas en la unidad experimental en $\mathrm{kg}$; $\mathrm{MS}=$ materia seca en fracción decimal; $\mathrm{G}=$ fracción de grano respecto al peso completo de mazorca; $\mathrm{FC}=$ factor de conversión para extrapolar el rendimiento de $5 \mathrm{~m}$ de surco a $12500 \mathrm{~m}$ que corresponde a la longitud total de surcos por hectárea a la separación de $0.8 \mathrm{~m}, 1.14$ es el factor para pasar el rendimiento de materia seca de grano a rendimiento con 14\% de humedad comercial.

Para identificar la productividad total del agua (PTA), se cuantificó la entrada artificial de agua por riego (AR) y se agregó el volumen total de agua de precipitación (AP) que se registró en la estación meteorológica del Servicio Meteorológico Nacional durante el ciclo del cultivo. Para conocer el valor de PTA se utilizó la expresión siguiente. $\mathrm{PTA}=\frac{\mathrm{RG}}{\mathrm{AT}}$.

Donde: $\mathrm{PTA}=$ productividad total del agua en $\mathrm{kg} \mathrm{m}^{-3} ; \mathrm{RG}=$ rendimiento de grano en $\mathrm{kg} \mathrm{ha}^{-1}$; AT= agua total utilizada por el cultivo para su evapotranspiración en $\mathrm{m}^{3} \mathrm{ha}^{-1}$; que también incluye las pérdidas por percolación y escurrimiento superficial, las cuales están en balance hidrológico con las entradas por riego y precipitación.

Para cuantificarla se registró el tiempo de riego y se aforó el caudal para los riegos en los ambientes correspondientes, también se llevó registro de la precipitación en la estación meteorológica de la FES Cuautitlán 0015043 y la 00015170 Chapingo, para identificar el volumen por esa variable durante el ciclo del cultivo. En este experimento no se estimó la lámina de riego, se observó el proceso que convencionalmente conducen los regadores en los dos ambientes bajo riego que, en conjunto con la precipitación, definieron la variante de disponibilidad de humedad.

\section{Resultados y discusión}

Se realizó el análisis de varianza revisando que se cumplieran los preceptos de la técnica bajo la hipótesis nula de la no existencia de efecto de los tratamientos sobre las variables respuesta. En el ANOVA la productividad total del agua (PTA) resultó con diferencias altamente significativas para ambientes (A) y para híbridos (B). 
Con diferencias significativas para la interacción ambiente $\mathrm{x}$ híbrido (A x B ) y ambiente x densidad (B x C). El coeficiente de variación para esta variable resultó de $17.7 \%$ y la media general de 0.99 $\mathrm{kg} \mathrm{m}^{-3}$ (Cuadro 1). La interacción entre factores indica que las respuestas observadas se deben a la combinación de sus efectos.

Cuadro 1. Cuadrados medios y significancia para productividad del agua, rendimiento y peso hectolítrico en tres ambientes de diez híbridos de maíz bajo dos densidades de siembra. Primavera-verano 2018.

\begin{tabular}{cccc}
\hline Fuente de variación & PTA $\left(\mathrm{kg} \mathrm{m}^{-3}\right)$ & $\mathrm{RG}\left(\mathrm{t} \mathrm{ha}^{-1}\right)$ & $\mathrm{PH}\left(\mathrm{kg} \mathrm{hl}^{-1}\right)$ \\
\hline Ambiente (A) & $2.65^{* *}$ & $22.31^{* *}$ & $516.34^{* *}$ \\
Híbrido (B) & $0.72^{* *}$ & $49.61^{* *}$ & $65.91^{* *}$ \\
Densidad (C) & 0.02 & 2.4 & 7.1 \\
A x B & $0.05^{*}$ & $4.17^{*}$ & 18.46 \\
A x C & 0.09 & $6.11^{*}$ & 5.02 \\
B x C & $0.06^{*}$ & $3.91^{*}$ & 8.12 \\
A x B x C & 0.02 & 1.26 & 14.84 \\
Media & 0.99 & 8.1 & 73.64 \\
CV (\%) & 17.7 & 17.3 & 4.89 \\
\hline
\end{tabular}

$*^{* * *}=$ significancia estadística al nivel del 0.05 y 0.01 , respectivamente; $\mathrm{CV}=$ coeficiente de variación; PTA= productividad total del agua $\left(\mathrm{kg} \mathrm{m}^{-3}\right) ; \mathrm{RG}=$ rendimiento de grano $\left(\mathrm{kg} \mathrm{ha}^{-1}\right) ; \mathrm{PH}=$ peso hectolítrico $\left(\mathrm{kg} \mathrm{hl}^{-1}\right)$.

La variable rendimiento de grano mostró diferencias altamente significativas en los factores de ambiente (A) e híbrido (B), también se encontró diferencia significativa en las interacciones ambiente $\mathrm{x}$ híbrido (A x B), ambiente $\mathrm{x}$ densidad ( $\mathrm{A} \times \mathrm{C}$ ) e híbrido $\mathrm{x}$ densidad ( $\mathrm{B}$ x $\mathrm{C}$ ), su coeficiente de variación fue de $17.3 \%$ y la media de $8.1 \mathrm{t} \mathrm{ha}^{-1}$ (Cuadro 1).

Se encontraron diferencias altamente significativas en el peso hectolítrico por el efecto de los factores ambiente (A) e híbrido (B), esta variable no mostró diferencia significativa del factor densidad de siembra (C) ni en las interacciones. Lo anterior muestra el efecto de los genotipos en la densidad del grano y el efecto de las condiciones edáficas y climáticas como también lo refiere Tadeo et al. (2014b).

En la interacción A x B se identificaron diferencias significativas en productividad del agua y rendimiento de grano. Estos resultados implican la respuesta diferenciada de los híbridos de acuerdo con los ambientes de modo que las variables de respuesta están influenciadas por las características edáficas y climáticas (Cuadro 1).

La interacción A x C sólo mostró diferencia significativa en rendimiento de grano y el porcentaje de variación con respecto a la media general de las variables de respuesta osciló entre 4.89 y 17.7\%. En la respuesta a los tratamientos generalmente tienen influencia las variables del clima, del suelo y del cultivo, cuando se manejan los mismos híbridos en ambientes diferentes puede existir interacción y son principalmente los dos primeros grupos de variables los que insertan la variabilidad en la respuesta, esta interpretación. 
Se ha observado en otros estudios como el de Martínez et al. (2018); Tadeo et al. (2014a) desarrollados con algunos materiales como los que se utilizaron en esta investigación. Debido a los resultados del Anova se realizó el análisis de medias para las interacciones de factores que resultaron con efecto significativo en las variables observadas.

La comparación de medias de la interacción ambiente $\mathrm{x}$ híbrido para la variable productividad total del agua resultó con diferencias estadísticas significativas como se muestra en el Cuadro 2, la interacción de los híbrido con el ambiente de la FESC-UNAM bajo temporal mostró las mayores PTA que en orden creciente $\left(0.64\right.$ a $1.22 \mathrm{~kg} \mathrm{~m}^{-3}$ ) fueron; H 51 AE, Ixim Puma, H 47 AE, Cuxi Puma, Tsíri Puma, H 45AE, H 53 AE, H-50, Tlaoli Puma y Atziri Puma.

Cuadro 2. Comparación de medias de las variables observadas de la interacción ambiente $x$ híbrido (A x B) del ciclo primavera-verano de 2018.

\begin{tabular}{|c|c|c|c|c|c|c|c|c|c|}
\hline \multirow{3}{*}{ Híbrido } & \multicolumn{3}{|c|}{ PTA $\left(\mathrm{kg} \mathrm{m}^{-3}\right)$} & \multicolumn{3}{|c|}{$\mathrm{RG}\left(\mathrm{t} \mathrm{ha}^{-1}\right)$} & \multicolumn{3}{|c|}{$\mathrm{PH}\left(\mathrm{kg} \mathrm{hl}^{-1}\right)$} \\
\hline & \multicolumn{2}{|c|}{ FESC-UNAM } & \multirow{2}{*}{$\frac{\text { Texcoco }}{\mathrm{R}}$} & \multicolumn{2}{|c|}{ FESC-UNAM } & \multirow{2}{*}{$\frac{\text { Texcoco }}{\mathrm{R}}$} & \multicolumn{2}{|c|}{ FESC-UNAM } & \multirow{2}{*}{$\begin{array}{c}\text { Texcoco } \\
\mathrm{R} \\
\end{array}$} \\
\hline & $\mathrm{T}$ & $\mathrm{R}$ & & $\mathrm{T}$ & $\mathrm{R}$ & & $\mathrm{T}$ & $\mathrm{R}$ & \\
\hline $\mathrm{H} 47 \mathrm{AE}$ & $0.91 \mathrm{bh}$ & $0.71 \mathrm{fl}$ & $0.56 \mathrm{kn}$ & $7.19 \mathrm{dh}$ & $6.68 \mathrm{fh}$ & $8.17 \mathrm{ag}$ & 73.4 af & 72.8 af & 74.5 af \\
\hline $\mathrm{H} 45 \mathrm{AE}$ & $1.04 \mathrm{ad}$ & $0.77 \mathrm{el}$ & $0.69 \mathrm{fl}$ & $8.19 \mathrm{ag}$ & $7.24 \mathrm{ch}$ & $10.06 \mathrm{ab}$ & $67.9 \mathrm{f}$ & $76 \mathrm{ae}$ & $77.7 \mathrm{ac}$ \\
\hline H $53 \mathrm{AE}$ & $1.12 \mathrm{ab}$ & $0.92 \mathrm{bh}$ & $0.58 \mathrm{jn}$ & 8.81 af & 8.71 af & $8.45 \mathrm{ag}$ & 73.4 af & 74.3 af & $78.3 \mathrm{ab}$ \\
\hline $\mathrm{H} 51 \mathrm{AE}$ & $0.64 \mathrm{im}$ & $0.33 \mathrm{n}$ & $0.41 \mathrm{mn}$ & $5.06 \mathrm{hi}$ & $3.12 \mathrm{i}$ & $5.97 \mathrm{gh}$ & $70.3 \mathrm{df}$ & $69.4 \mathrm{df}$ & 72.5 af \\
\hline H-50 & $1.14 \mathrm{ab}$ & $0.79 \mathrm{dk}$ & $0.67 \mathrm{gm}$ & 8.98 af & $7.44 \mathrm{bh}$ & $9.86 \mathrm{ac}$ & $68.6 \mathrm{f}$ & $71.5 \mathrm{bf}$ & 74.5 af \\
\hline Tsíri Puma & $0.97 \mathrm{ae}$ & $0.91 \mathrm{bh}$ & $0.56 \mathrm{kn}$ & $7.67 \mathrm{bh}$ & $8.59 \mathrm{ag}$ & $8.23 \mathrm{ag}$ & 73.9 af & $76.6 \mathrm{ad}$ & $79.2 \mathrm{a}$ \\
\hline Tlaoli Puma & $1.15 \mathrm{ab}$ & $0.93 \mathrm{bg}$ & $0.66 \mathrm{hm}$ & 9.06 af & 8.78 af & $9.73 \mathrm{ad}$ & $71.8 \mathrm{bf}$ & 72.8 af & $77.6 \mathrm{ac}$ \\
\hline Atziri Puma & $1.22 \mathrm{a}$ & $1.06 \mathrm{ac}$ & $0.73 \mathrm{el}$ & $9.61 \mathrm{ae}$ & $9.98 \mathrm{ab}$ & $10.74 \mathrm{a}$ & 72.8 af & 73.2 af & $76.3 \mathrm{ad}$ \\
\hline Ixim Puma & $0.89 \mathrm{bi}$ & $0.83 \mathrm{cj}$ & $0.51 \ln$ & $7.02 \mathrm{eh}$ & $7.85 \mathrm{bg}$ & $7.47 \mathrm{bh}$ & $71.1 \mathrm{cf}$ & 72.7 af & $76.3 \mathrm{ad}$ \\
\hline Cuxi Puma & $0.95 \mathrm{bf}$ & $0.89 \mathrm{bi}$ & $0.56 \mathrm{kn}$ & $7.49 \mathrm{bh}$ & $8.41 \mathrm{ag}$ & $8.2 \mathrm{ag}$ & $71.1 \mathrm{cf}$ & $71.9 \mathrm{bf}$ & $77.4 \mathrm{ac}$ \\
\hline DMS & & 0.26 & & & 2.63 & & & 6.77 & \\
\hline
\end{tabular}

Las medias con la misma letra dentro del grupo de medias de las variables observadas son iguales estadísticamente (Tukey, $p=0.05$ ); $\mathrm{T}=$ temporal; $\mathrm{R}=$ riego; $\mathrm{DMS}=$ diferencia mínima significativa; $\mathrm{PTA}=$ productividad total del agua; $\mathrm{RG}=$ rendimiento de grano; $\mathrm{PH}=$ peso hectolítrico.

En esta interacción el volumen total por lluvia y riego fue $7888 \mathrm{~m}^{3} \mathrm{ha}^{-1}$, al ingresar menor cantidad de agua al sistema y generar rendimientos incluso superiores a la interacción con el ambiente FESC-UNAM bajo riego, se incrementa el indicador de productividad como ha resultado en otros estudios (FAO, 2002; Rivetti, 2006; Zamora et al., 2011; Saenz et al., 2014) en algunos trabajos se atribuye el contraste en las respuestas a la interacción con las condiciones edáficas y climáticas de los ambientes (Zamudio et al., 2015; Canales et al., 2017; López et al., 2017; Martínez et al., 2018).

La interacción de los ambientes con los híbridos Atziri Puma y Tlaoli Puma generados por la UNAM en su esquema de mejoramiento de maíz (Tadeo et al., 2016a), mostraron la productividad más elevada siendo una medida indirecta del uso eficiente del agua por lo que, a reserva de otras investigaciones sobre variables hídricas, estos híbridos tienen potencial productivo en los ambientes estudiados de los Valles Altos donde la disponibilidad del agua es limitada. 
El híbrido H 51 AE en su interacción con los ambientes mostró menor productividad total del agua (Cuadro 2), lo generó el INIFAP (Espinosa et al., 2012) y su uso se debe reservar a las condiciones donde el recurso agua no es limitante. Se observa también que las versiones de híbridos androestériles (AE), referidos en otros trabajos de Espinosa et al. (2009); Tadeo et al. (2014b), en la interacción con los ambientes mostraron productividad del agua baja que según Tadeo et al. (2014a) para otras variables agronómicas de rendimiento puede estar relacionada con la coincidencia de las líneas que sintetizan su estructura híbrida.

La interacción del híbrido Atziri Puma con los ambientes FESC-UNAM temporal y Texcoco mostró la productividad del agua y rendimiento superior respectivamente. Lo anterior coincide con lo encontrado en otros trabajos (Tadeo et al., 2016b; Martínez et al., 2018) donde se observaron respuestas similares, estos resultados muestran la oportunidad de ofrecer a los productores una alternativa de maíz híbrido en los ambientes estudiados con ventajas económicas y agronómicas competitivas.

En el ambiente de la FESC-UNAM bajo temporal el volumen de agua por lluvia fue de $7888 \mathrm{~m}^{3}$ $\mathrm{ha}^{-1}$, en FESC-UNAM bajo riego fueron $7888 \mathrm{~m}^{3} \mathrm{ha}^{-1}$ de lluvia más $1548 \mathrm{~m}^{3} \mathrm{ha}^{-1}$ de riego en Texcoco bajo riego fueron $7328 \mathrm{~m}^{3} \mathrm{ha}^{-1}$ de lluvia y $7372.8 \mathrm{~m}^{3} \mathrm{ha}^{-1}$ de riego. Según los datos anteriores, en el Cuadro 2 se observa que la PTA decrece cuando se incrementa el volumen total de agua utilizado lo que indica una relación inversa.

Los promedios de PTA y RG de las interacciones A x B en el ambiente de la FESC-UNAM bajo temporal fueron superiores con respecto al ambiente FESC-UNAM bajo riego. Esta diferencia puede deberse a las condiciones de disponibilidad de humedad por el desfase entre las fechas de siembra, el ensayo de temporal se inició una semana después con el temporal establecido disponiendo de agua en cantidad y oportunidad, estos resultados contrastan a los reportados para otros estudios donde se aporta el riego suplementario en cantidad según el requerimiento (Rivetti, 2006; Avendaño et al., 2008; Saenz et al., 2014).

Sin embargo, cuando se agrega el total de los volúmenes requeridos, no siempre coincide con el mayor rendimiento y productividad del agua, ya que cuando la eficiencia de riego es baja, se pierden volúmenes por escurrimiento o por percolación profunda. La justificación del porque la interacción de los híbridos con el ambiente de la FESC-UNAM bajo riego mostró menor productividad total del agua que la interacción con FESC-UNAM bajo temporal.

Se explica también con los indicadores de eficiencia en el manejo del agua a nivel parcelario ya que la eficiencia de aplicación del requerimiento (Ear) en una evaluación resultó de 76\%, la eficiencia de aplicación del riego (Ea) de $70 \%$ y el coeficiente de uniformidad de Christiansen (CUC) de $85 \%$. Esto indica que la mala distribución del agua incide en el aprovechamiento por el cultivo que implica un desperdicio de agua traduciendo su efecto al rendimiento.

Una variable operativa del riego que marca la respuesta al rendimiento es la disponibilidad de humedad en el tiempo oportuno (Palacios, 2007) por lo que, un día de estrés por debajo del punto crítico reduce significativamente el rendimiento (Allen et al., 2006; Ojeda et al., 2015). Otro aspecto relacionado con el efecto del riego es el cambio en la densidad aparente por el ciclo de saturación y secado del suelo que es más severo en suelo con alto contenido de arcilla (González et al., 2009; Madero et al., 2012). 
Teniendo un efecto en el desarrollo inicial y posiblemente en las variables respuesta. En esas condiciones para la región de influencia de la FESC-UNAM se recomienda manejar con pericia el riego de punteo con los híbridos estudiados cuando se tengan registros históricos de buen temporal y disponibilidad de información que permita sincronizar la siembra con el periodo de lluvias.

En cuanto al rendimiento la interacción del ambiente con el híbrido Atziri Puma mostró el valor estadísticamente superior en Texcoco $\left(10.74 \mathrm{t} \mathrm{ha}^{-1}\right)$ y el más bajo $\left(3.12 \mathrm{t} \mathrm{ha}^{-1}\right)$ en el H $51 \mathrm{AE}$ en la FESC-UNAM bajo riego, en el primer caso hay coincidencia con los estudios donde se ha encontrado relación directa entre la cantidad de agua aportada y el rendimiento (Zamora et al., 2011; López et al., 2019).

La interacción de los híbridos con los ambientes de la FESC-UNAM muestra diferencias significativas en PTA y RG, por lo que se requieren estudios precisos sobre el efecto del riego de punteo con relación al temporal ya que el aporte de agua en cantidad y oportunidad impacta en el rendimiento como lo refiere Allen et al. (2006); Ojeda et al. (2015).

La interacción de los híbridos del INIFAP con el ambiente bajo temporal tiene buena respuesta en el rendimiento (Cuadro 2), por ello evitando el riego de punteo en los híbridos estudiados se ahorra agua, en este caso $1548 \mathrm{~m}^{3} \mathrm{ha}^{-1}$, que se puede destinar a otros cultivos incrementando la productividad marginal o se puede dejar almacenada en la fuente de abastecimiento para utilizarla como riego de auxilio cuando se agrava la sequía interestival a finales de julio y principios de agosto. Aunque el riego de punteo es una actividad que asegura el establecimiento de la siembra, debe manejarse con técnica cuando el agua es un recurso limitado.

En el Cuadro 3 se muestra la interacción ambiente x densidad de siembra donde se observó que la productividad total del agua se reduce conforme se incrementa la cantidad de agua suministrada y no tiene efecto significativo el incremento de la densidad de siembra, la condición de disponibilidad de humedad es significativa y la densidad de siembra no. En el caso de la variable de rendimiento se observa interacción significativa de la disponibilidad de humedad y de la densidad de siembra.

Cuadro 3. Comparación de medias para las variables observadas de la interacción ambiente x densidad de siembra (A x C). Ciclo primavera-verano de 2018.

\begin{tabular}{|c|c|c|c|c|c|c|c|c|c|}
\hline \multirow{3}{*}{$\begin{array}{c}\text { Densidad } \\
\left(\text { plantas }^{-1} \mathrm{ha}^{-1}\right)\end{array}$} & \multicolumn{3}{|c|}{ PTA $\left(\mathrm{kg} \mathrm{m}^{-3}\right)$} & \multicolumn{3}{|c|}{$\mathrm{RG}\left(\mathrm{t} \mathrm{ha}^{-1}\right)$} & \multicolumn{3}{|c|}{ PH $\left(\mathrm{kg} \mathrm{hl}^{-1}\right)$} \\
\hline & \multicolumn{2}{|c|}{ FESC-UNAM } & \multirow{2}{*}{$\frac{\text { Texcoco }}{\mathrm{R}}$} & \multicolumn{2}{|c|}{ FESC-UNAM } & \multirow{2}{*}{$\frac{\text { Texcoco }}{\mathrm{R}}$} & \multicolumn{2}{|c|}{ FESC-UNAM } & \multirow{2}{*}{$\frac{\text { Texcoco }}{\mathrm{R}}$} \\
\hline & $\mathrm{T}$ & $\mathrm{R}$ & & $\mathrm{T}$ & $\mathrm{R}$ & & $\mathrm{T}$ & $\mathrm{R}$ & \\
\hline 65000 & $0.97 \mathrm{a}$ & $0.78 \mathrm{~b}$ & $0.61 \mathrm{c}$ & $7.68 \mathrm{bc}$ & $7.39 \mathrm{c}$ & $8.91 \mathrm{a}$ & $71.77 \mathrm{~b}$ & $73.01 \mathrm{~b}$ & $76.66 \mathrm{a}$ \\
\hline 80000 & $1.03 \mathrm{a}$ & $0.84 \mathrm{~b}$ & $0.58 \mathrm{c}$ & $8.14 \mathrm{ac}$ & $7.97 \mathrm{bc}$ & $8.47 \mathrm{ab}$ & $71.03 \mathrm{~b}$ & $73.23 \mathrm{~b}$ & $76.14 \mathrm{a}$ \\
\hline DMS & & 0.09 & & & 0.894 & & & 2.299 & \\
\hline
\end{tabular}

Las medias con la misma letra dentro del grupo de medias de las variables observadas son iguales estadísticamente (Tukey, $p=0.05$ ); $\mathrm{T}=$ temporal; $\mathrm{R}=$ riego; $\mathrm{DMS}=$ diferencia mínima significativa; $\mathrm{PTA}=$ productividad total del agua; $\mathrm{RG}=$ rendimiento de grano; $\mathrm{PH}=$ peso hectolítrico.

Estos resultados son similares a los reportados por Virgen et al. (2016); Canales et al. 2017) quienes señalan que el rendimiento crece al incrementar la densidad de población, así también Espinosa et al. (2012) menciona que los genotipos tienen buena respuesta al incremento en la densidad de población. 
Las medias de la interacción híbrido x densidad de siembra se muestra en el Cuadro 4 y se observa diferencias significativas en la productividad total del agua conforme varían los híbridos en su interacción con las densidades de siembra resultando valores entre 0.44 y $1.01 \mathrm{~kg} \mathrm{~m}^{-3}$, el efecto en las variables respuesta que insertan los híbridos es significativa y la densidad de siembra también.

Cuadro 4. Comparación de medias de las variables observadas de la interacción híbrido $\mathrm{x}$ densidad de siembra (B x C). Ciclo primavera-verano de 2018.

\begin{tabular}{|c|c|c|c|c|c|c|}
\hline \multirow{3}{*}{ Híbrido } & \multicolumn{2}{|c|}{ PTA $\left(\mathrm{kg} \mathrm{m}^{-3}\right)$} & \multicolumn{2}{|c|}{$\mathrm{RG}\left(\mathrm{t} \mathrm{ha}^{-1}\right)$} & \multicolumn{2}{|c|}{$\mathrm{PH}$} \\
\hline & \multicolumn{6}{|c|}{ Densidad de siembra (plantas ha ${ }^{-1}$ ) } \\
\hline & 65000 & 80000 & 65000 & 80000 & 65000 & 80000 \\
\hline $\mathrm{H} 47 \mathrm{AE}$ & $0.65 \mathrm{de}$ & $0.8 \mathrm{ad}$ & $6.56 \mathrm{de}$ & $8.13 \mathrm{ad}$ & $73.2 \mathrm{ac}$ & $73.9 \mathrm{ac}$ \\
\hline $\mathrm{H} 45 \mathrm{AE}$ & $0.81 \mathrm{ad}$ & $0.86 \mathrm{ac}$ & $8.25 \mathrm{ad}$ & $8.74 \mathrm{ac}$ & $75.2 \mathrm{ab}$ & $72.6 \mathrm{ac}$ \\
\hline H 53AE & $0.81 \mathrm{ad}$ & $0.93 \mathrm{ab}$ & $8.14 \mathrm{ad}$ & $9.17 \mathrm{ab}$ & $75.3 \mathrm{ab}$ & $75.3 \mathrm{ab}$ \\
\hline $\mathrm{H} 51 \mathrm{AE}$ & $0.44 \mathrm{e}$ & $0.48 \mathrm{e}$ & $4.62 \mathrm{e}$ & $4.81 \mathrm{e}$ & $71.8 \mathrm{bc}$ & $69.6 \mathrm{c}$ \\
\hline $\mathrm{H}-50$ & $0.9 \mathrm{ac}$ & $0.84 \mathrm{ad}$ & $9.16 \mathrm{ab}$ & $8.37 \mathrm{ad}$ & $71.6 \mathrm{bc}$ & $71.4 \mathrm{bc}$ \\
\hline Tsíri Puma & $0.77 \mathrm{bd}$ & $0.86 \mathrm{ac}$ & $7.69 \mathrm{bd}$ & $8.64 \mathrm{ac}$ & $76 \mathrm{ab}$ & $77.1 \mathrm{a}$ \\
\hline Tlaoli Puma & $0.92 \mathrm{ac}$ & $0.91 \mathrm{ac}$ & $9.23 \mathrm{ab}$ & $9.15 \mathrm{ab}$ & $73.7 \mathrm{ac}$ & $74.3 \mathrm{ac}$ \\
\hline Atziri Puma & $1.01 \mathrm{a}$ & $1 \mathrm{a}$ & $10.15 \mathrm{a}$ & $10.08 \mathrm{a}$ & $74.3 \mathrm{ac}$ & $73.8 \mathrm{ac}$ \\
\hline Ixim Puma & $0.77 \mathrm{bd}$ & $0.72 \mathrm{~cd}$ & $7.88 \mathrm{bd}$ & $7.01 \mathrm{~cd}$ & $73.5 \mathrm{ac}$ & $73.3 \mathrm{ac}$ \\
\hline Cuxi Puma & $0.81 \mathrm{ad}$ & $0.79 \mathrm{bd}$ & $8.24 \mathrm{ad}$ & $7.83 \mathrm{bd}$ & $73.7 \mathrm{ac}$ & $73.2 \mathrm{ac}$ \\
\hline DMS & \multicolumn{2}{|c|}{0.205} & \multicolumn{2}{|c|}{2.031} & \multicolumn{2}{|c|}{5225} \\
\hline
\end{tabular}

Las medias con la misma letra dentro del grupo de medias de las variables observadas son iguales estadísticamente (Tukey, $p=0.05$ ); DMS= diferencia mínima significativa; PTA= productividad total del agua; $\mathrm{RG}=$ rendimiento de grano; $\mathrm{PH}=$ peso hectolítrico.

La interacción de Atziri Puma con las dos densidades de siembra muestra la mayor productividad, pero no hay diferencia significativa entre ellas lo mismo ocurre con el rendimiento. En particular para esta interacción convendría utilizar la densidad de siembra más baja que reduce el costo de adquisición de semilla. La interacción del H 51AE con las densidades de siembra produce los promedios más bajos de productividad y de rendimiento sin mostrar diferencia significativa.

En el caso de la variable de rendimiento se observa interacción significativa de los híbridos con la densidad de siembra mostrando valores que varían entre 4.62 y $10.15 \mathrm{t} \mathrm{ha}^{-1}$. Los híbridos de INIFAP a excepción del H 51 AE muestran rendimiento mayor al interaccionar con la densidad de 80000 plantas ha ${ }^{-1}$, los híbridos UNAM a excepción del Tsíri Puma muestran rendimiento superior en su interacción con la densidad de 65000 plantas ha $^{-1}$.

\section{Conclusiones}

Se identificó la productividad total del agua promedio $\left(0.99 \mathrm{~kg} \mathrm{~m}^{-3}\right)$, el rendimiento de grano promedio $\left(10.1 \mathrm{t} \mathrm{ha}^{-1}\right)$ y el peso hectolítrico promedio $\left(73.64 \mathrm{~kg} \mathrm{hl}^{-1}\right)$ en tres ambientes diferenciados por la disponibilidad de humedad (factor A) de 10 híbridos de maíz blanco (factor B) bajo dos densidades de siembra (factor C), se encontraron interacciones A x B y B x C en PTA y A x B, A x C, B x C en RG. En el peso hectolítrico se encontraron diferencias significativas por el efecto de los factores A y B que implican la manifestación de los fenotipos y genotipos. 
La productividad total del agua está relacionada inversamente con la cantidad de agua que ingresa al sistema de producción, los valores encontrados son indicadores de la baja eficiencia en el uso del agua en las interacciones de los ambientes donde se suministró riego. Se recomienda realizar las estimaciones del requerimiento de riego en tiempo real y transferirlas a través de asesoría técnica a los agricultores.

EL híbrido Atziri Puma mostró mayor productividad total del agua y rendimiento de grano en su interacción con los ambientes y no tuvo diferencia significativa en la interacción con la densidad de siembra. Este híbrido tiene potencial para utilizarse en los lugares con características similares a las de este estudio, donde el agua es un recurso limitado y por lo tanto valioso por su escasez.

\section{Agradecimientos}

Esta investigación fue parte del programa de apoyo a proyectos de investigación e innovación tecnológica PAPIIT: IT201618, UNAM.

\section{Literatura citada}

Allen, R. G; Pereira, S. L.; Raes, D. y Smith, M. 2006. Evapotranspiración del cultivo: guías para la determinación de los requerimientos de agua de los cultivos. FAO. Primera edición. Roma, Italia. Boletin 56. 277 pp.

Avendaño, A. C.; Molina, G. J.; Trejo, L. C.; López, C. C. y Cadena, I. J. 2008. Respuesta a altos niveles de estrés hídrico en maíz. Agron. Mesoam. 19(1):27-37.

Canales, I. E. I.; Tadeo, R. M.; Mejía, C. J. A.; García, Z. J. J. y Espinosa, C. A. 2017. Semilla fértil y androestéril de maíz bajo diferentes densidades de población. Ecosist. Recur. Agropec. 12(4):465-473.

CONAGUA. 2014. Curso de capacitación: diseño, implementación y seguimiento del riego por gravedad. México, DF. 204 p.

Espinosa, C. A.; Tadeo, R. M.; Gómez, M. N.; Sierra, M. M.; Martínez, M. R.; Virgen, V. J.; Palafox, C. A.; Caballero, H. F.; Vázquez, C. G. y Salinas, M. Y. 2008. H-49 AE híbrido de maíz para Valles Altos con androestérilidad para producción de semilla. In: Día de Campo CEVAMEX. Memoria técnica núm 9. Chapingo, Estado de México. 13-14 pp.

Espinosa, C. A.; Tadeo, R. M.; Sierra, M. M.; Turrent, F. A.; Valdivia, B. R. y Zamudio, G. B. 2009. Rendimiento de híbridos de maíz bajo diferentes combinaciones de semilla androestéril y fértil en México. Agron. Mesoam. 20(2):211-216.

Espinosa, C. A.; Tadeo, R. M.; Virgen, V. J.; Rojas, M. I.; Gómez, M. N. O.; Sierra M. M.; Palafox, C. A.; Vázquez, C. G.; Rodríguez, M. F. A.; Zamudio, G. B.; Arteaga, E. I.; Canales, I. E.; Martínez, Y. B. y Valdivia, B. R. 2012. H-51 AE, híbrido de maíz para áreas de humedad residual, buen temporal y riego en Valles Altos del Centro de México. Rev. Fitotec. Mex. 35(4):347-349.

Espinosa, C. A.; Tadeo, R. M.; Virgen, V. J.; Zamudio, G. B.; Turrent, F. A. y López, L. C. 2019. H 47AE, H 49AE y H 53AE híbridos de maíz con androesterilidad para Valles Altos. Primer Simposio para la Autosuficiencia y Soberania Alimentaria de México. V Reunión Estatal de Investigación. Publicación especial núm. 1, Campo Experimental Valle de México, INIFAP. Texcoco, Estado de México. 8-12 pp. 
Espinosa, C. A.; Tadeo, R. M.; Zamudio, G. B.; Virgen, V. J.; Turrent, F. A.; Rojas, M. I.; Gómez, M. N. O.; Sierra, M. M.; López, L. C.; Palafox, C. A.; Vázquez, C. G.; Rodríguez, M. F.; Canales, I. E.; Zaragoza, E. J.; Martínez, Y. B.; Valdivia, B. R.; Cárdenas, M. A.; Mora, G. K. y Martínez, N. B. 2018. H-47 AE, híbrido de maíz para Valles Altos de México. Rev. Fitotec. Mex. 1(41):87-89.

FAO-AGL. 2002. Maize crop water management FAOSTAT, 2003. http://faostat.fao.org/faostat/ fo rm?collection=Production. Crops.Primary\&Dom ain.

FAO. 2002. Agricultura mundial: hacia los años 2015-2030. http://www.fao.org/docrep/ 004/y3557s/y3557s0 0.htm\#TopOfPage.

González, C. O.; Iglesias, C. C. y Herrera, S. M. 2009. Análisis de los factores que provocan compactación del suelo agrícola. Rev. Cienc. Téc. Agropec. 18(2):57-63.

González, E. A.; Islas, G. A.; Espinosa C. A.; Vázquez, C. A. y Wood S. 2007. Impacto económico del mejoramiento genético del maíz en México: híbrido H-50. Publicación especial núm. 24. INIFAP. Texcoco, Estado de México. 83 p.

González, E. A.; Islas, G. A.; Espinosa C. A.; Vázquez, C. A. y Wood S. 2008. Impacto económico del mejoramiento genético del maíz en México: híbrido H-48. Publicación especial núm. 24. INIFAP. Texcoco, Estado de México. 83 p.

INIFAP. 1988. Instituto Nacional de Investigaciones Forestales, Agrícolas y Pecuarias. Paquetes tecnológicos de cultivos. Campo Experimental del Valle de Santo Domingo. SAGARINIFAP-CIRNO, México. 75-81 p.

Lamm, F. R. y Trooien, T. P. 2001. Irrigation capacity and plant population effects on corn production using SDI. In Proc. Irrigation Assessment. Irrigation technical Conference. Primera edición. Falls Church VA. San Antonio, Texas. 73-80 p.

López, H. M.; Arteaga, R. R.; Ruiz, G. A.; Vázquez, P. M. y López, R. J. 2019. Productividad del agua normalizada para maíz (Zea mays) en Chapingo, México. Agrociencia. 53(6):811-820.

López, L. C.; Tadeo, R. M.; Espinosa, C. A.; García, Z. J.; Benítez, R. I.; Vázquez, C. M. y Carrillo, S. J. 2017. Productividad de cruzas simples de maíz con calidad de proteína en Valles Altos de México. México. Rev. Mex. Cienc. Agríc. 8(3):559-570.

Madero, M. E.; Peña A. M.; Escobar, B. Y.; García, L. F. 2012. Compactación potencial en dos suelos de la parte plana del Valle del Cauca. Acta Agronómica. 61(1):27-31.

Martínez, G. A.; Zamudio, G. B.; Tadeo, R. M.; Espinosa, C. A.; Cardoso, G. J.; Vázquez, C. G. y Turrent, F. A. 2018. Rendimiento de híbridos de maíz grano blanco en cinco localidades de Valles Altos de México. Rev. Mex. Cienc. Agríc. 9(7):1447-1458.

Ojeda, B. W. y Flores, V. J. 2015. Calendarización del riego: teoría y práctica. IMTA. Jiutepec, Morelos, México. 295 p.

Palacios, V. E. 2007. La operación de los sistemas de riego con las técnicas de la información. Chapingo, Estado de México. Primera edición. México, DF. 299 p.

Rivetti, A. R. 2006. Producción de maíz bajo diferentes regímenes de riego complementario en Río Cuarto, Córdoba, Argentina. I. Rendimiento en grano de maíz y sus componentes. Rev. Fac. Cienc. Agrar. 38(2):25-36.

Saenz, C. A.; Gómez, H. V.; Frigerio, K. L.; Morábito, J. A.; Terenti, O. A. y Cortes, M. P. 2014. Rendimiento de grano y eficiencia en el uso del agua en maíz bajo riego complementario con agua salina. Rev. Investig. Agropec. 40(3):252-259.

Tadeo, R. M.; Espinosa, C. A.; García, Z. J. J.; Lobato, O. R.; Gómez, M. N. O.; Sierra M. M.; Valdivia, B. R.; Zamudio, G. B.; Martínez, Y. B.; López, L. C.; Mora, G. K. Y.; Canales, I. E. I.; Cárdenas, M. A. L.; Zaragoza, E. J. y Alcántar, L. H. J. 2016a. Tsiri Puma, híbrido de maíz para Valles Altos con esquema de androesterilidad para producción de semillas. Rev. Fitotec. Mex. 39(3):331-333. 
Tadeo, R. M.; Espinosa, C. A.; Turrent, F. A.; Zamudio, G. B.; Sierra, M. M.; Gómez, M. N.; Valdivia, B. R. y Virgen, V. J. 2014b. Rendimiento de híbridos androestériles y fértiles de maíz en dos localidades de Valles Altos de México. Revi. Mex. Cienc. Agríc. 5(5):883-891.

Tadeo, R. M.; Espinosa, C. A.; Turrent, F. A.; Zamudio, G. B.; Valdivia, B. R. y Andrés, M. P. 2014a. Productividad de grano de cuatro híbridos trilineales de maíz en versión androesteril y fértil. Agron. Mesoam. 25(1):45-52.

Tadeo, R. M.; Zaragoza, E. J.; Espinosa, C. A.; Turrent, F. A.; Zamudio, G. B.; Virgen, V. J.; Mora, G. K. Y. y Valdivia, B. R. 2016b. Productividad de la generación F1 y F2 de híbridos de maíz (Zea mays L.) de Valles Altos de México. Agrociencia. 50(1):33-41.

Virgen, V. J.; Zepeda, B. R.; Ávila, P. M. A.; Espinosa, C. A.; Arellano, V. J. L. y Gámez, V. A. 2016. Producción y calidad de semillas de maíz en Valles Altos de México. Agron. Mesoam. 27(1):191-206.

Zamora, S. S.; Ruíz, E. I.; Beltrán, M. F.; Fenech, L. L.; Murillo, A. B.; Loya, R. J. y Troyo, D. E. 2011. Régimen hídrico del maíz en una zona árida, determinado en porcentajes de evaporación. Trop. Subtrop. Agroecosys. 13(1):181-186.

Zamudio, G. B.; Tadeo, R. M.; Espinosa, C. A.; Martínez, R. J. N.; Celis, E. D. I. y Valdivia, B. R. 2015. Eficiencia agronómica de fertilización al suelo de macronutrimentos en híbridos de maíz. México. Rev. Mex. Cienc. Agríc. 6(7):1557-1569. 